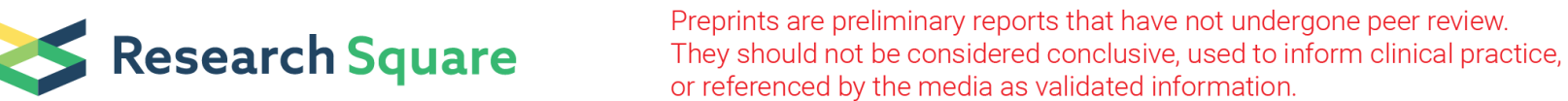

\section{The Mechanism of Mitochondrial Fatty Acid Oxidation's Involvement in Osteoporosis in Aggravating Cartilage Degeneration of Rabbit Knee Osteoarthritis}

\section{Ying Jia}

The First Affiliated Hospital of Chongqing Medical University

\section{Ziting Deng ( $\square$ denry_beta@hospital.cqmu.edu.cn )}

Chongqing University of Medical Science: Chongqing Medical University https://orcid.org/0000-00019209-999X

\section{Li Wen}

The First Affiliated Hospital of Chongqing Medical University

\section{Li Zeng}

The First Affiliated Hospital of Chongqing Medical University

\section{Yanhua Chen}

The Affiliated Rehabilitation Hospital of Chongqing Medical UniversityON

\section{Research article}

Keywords: knee osteoarthritis, Osteoprotegerin, osteoprotegerin ligand, interleukin $1 \beta$, carnitine palmitoyl transferase I B

Posted Date: February 19th, 2021

DOI: https://doi.org/10.21203/rs.3.rs-216060/v1

License: (c) (i) This work is licensed under a Creative Commons Attribution 4.0 International License. Read Full License 


\section{Abstract}

Objective: To observe whether the oxidative metabolism of mitochondrial fatty acids is involved in osteoporosis by detecting the changes in the contents of interleukin $1 \beta$, osteoprotegerin (OPG) and

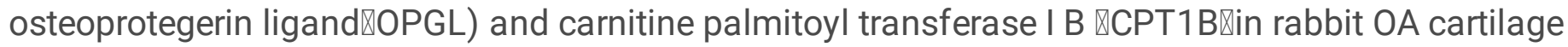
Regulation of experimental rabbit knee OA.

Methods: 30 female New Zealand rabbits were randomly divided into 3 groups with 10 in each group: Normal group ,OP+OA group and OA group. After successful modeling, the knee articular cartilage of each group were taken; The BMC and BMD of each group were measured; Observe the results of $\mathrm{HE}$ staining of cartilage; immunohistochemical method Detection of IL-1 $\beta$; The Q-PCR method and Western blot method were used to detect IL-1 $\beta$, OPG, OPGL and CPT1B mRNA and protein expression in cartilage.

Results: Compared with the normal group, the expression of IL-1 $\beta$, OPGL and CPT1B in the OP+OA group and the OA group was significantly increased $(P<0.01)$, while the expression of OPG decreased $(P<0.01)$; Compared with OP+OA group, The expression of OPG and the ratio of OPG/OPGL was significantly higher in the OA group $(P<0.01)$. Compared with OA group, The expression of IL-1 $\beta$, OPGL and CPT1B in the $\mathrm{OP}+\mathrm{OA}$ group was significantly higher $(\mathrm{P}<0.01)$;

Conclusion: Mitochondrial fatty acid oxidation metabolism can be involved in the effect of osteoporosis on osteoarthritis by up-regulating the expression of CPT1b, which can mediate changes in the OPG/OPGL ratio, increase the expression of IL-1 $\beta$, and aggravate the Degeneration of OA cartilage and subchondral bone.

\section{Introduction}

Osteoarthritis (OA) is a multifactorial chronic disease characterized by damage to tissues around joints, especially cartilage destruction, loss and abnormal changes in subchondral bone.[1] Osteoarthritis (OA) and osteoporosis (OP) are two common musculoskeletal disorders that cause disability and reduce the quality of life.[2]

Recently, the Osteoarthritis Research Society International (OARSI) found that OA are associated with low bone volume or osteoporosis, as evidenced by a reduction in bone density under specific cartilage caused by systemic or local OP, accompanied by high bone remodeling rates.[3]. Osteoporotic osteoarthritis (OPOA) is a special form of OA, and it is mainly associated with brittle subchondral bone and micro fracture, both of which accelerate OA progress. Subchondral bone and the synovium play an important role in the onset and progression of $\mathrm{OA},[1]$,which is located in the osteopathic area below the joint cartilage, consists of the subchondral bone plate and Cancellous bone. The subchondral bone ultrastructure in the osteoporotic OA model was characterized by the destruction of the network structure and collagen fibers. The subchondral bone ultrastructural damage caused by osteoporosis may change mechanical properties of the upper cartilage and aggravate OA cartilage[4]. The OPG-RANKL-RANK interplay in balancing the resorption and remodeling of subchondral bone[5]. OPG is protective in the 
cartilage[6], while RANKL and a high RANKL/OPG ratio are associated with a pro-inflammatory state, leading to destruction of cartilage tissue via activation of MMP-13.

Mitochondrial fatty acid $\beta$-oxidation is the major pathway for the catabolism of fatty acids, and it plays an essential role in maintaining whole body energy homeostasis. Studies have found that the CPT1A gene is associated with osteoporosis[7]. Therefore, we hypothesized that fatty acid oxidation is involved in the pathogenesis of OPOA. To test the hypothesis, by detecting the expression changes of IL-1 $\beta$, OPG, OPGL and CPT1B in articular cartilage, we explored whether osteoporosis is involved in the damage mechanism of osteoarthritis by regulating the expression of CPT1B, OPG \OPGL ratio and IL-1ß.

\section{Material And Methods}

\subsection{ANIMAIS}

Thirty 4-months-old healthy adult female New Zealand rabbits weighing 2.0-2.5KG were provided by the Animal Experimental Center of Chongqing Medical University. Animals were housed in individual cages with a $12: 12$-h light-dark cycle at $20-26^{\circ} \mathrm{C}$, and received a standard laboratory diet and drinking water. Laboratory animal production license number: SCXK (Chongqing) 2017-0001.

\subsection{Experimental design}

30 female New Zealand rabbits were randomly divided into 3 groups with 10 in each group: normal group, $\mathrm{OP}+\mathrm{OA}$ group, and $\mathrm{OA}$ group. The $\mathrm{OA}$ group experienced $\mathrm{OA}$ surgery on right knees, the $\mathrm{OP}+\mathrm{OA}$ group $\mathrm{OA}$ surgery and ovariectomy. After BMD/BMC measurement, all animals were killed in 4 weeks after $\mathrm{OA}$ surgery.

\section{3| Ovariectomy}

The rabbits were anesthetized by intraperitoneal injection of $0.035 \mathrm{~g} / \mathrm{kg}$ chloral hydrate. After the anesthesia, the rabbits were fixed on the operating table in prone position. With routine skin preparation, routine disinfection and draping, we entered the abdominal cavity of rabbits through the middle and lower abdominal incision, and removed both ovaries, and carefully stopped the bleeding. After cleaning, we sutured the muscle, subcutaneous tissue and skin layer by layer[8].

\subsection{OA surgery}

Under aseptic conditions, an incision of about $2 \mathrm{~cm}$ was made along the inner side of the right knee joint to expose the knee joint to cut off the anterior and posterior cruciate ligaments and medial collateral ligament, and completely resect the medial meniscus, suture the joint cavity layer by layer[6]. After operation, penicillin was injected intramuscularly at $2 \times 104 \mathrm{U} / \mathrm{kg}$ every day for one week, and the rabbits were driven to run for 30 minutes every day for 4 weeks.

2.5 Tissue sampling and morphological testing 
Take the rabbits' right knee articular cartilage platform and subchondral bone. The tissue is divided into two parts. One part was put into the marked EP tube and immediately placed in a liquid nitrogen tank for quick freezing, and then transferred to $-80^{\circ} \mathrm{C}$ for storage in the refrigerator for testing; another part is fixed in $4 \%$ paraformaldehyde solution and embedded.

The general conditions of rabbit synovium, joint capsule, joint fluid and articular cartilage in each group, and Pelletier-JP [9] standard score were visually observed and recorded; Scoring criteria: 0 is the articular surface is smooth and the color is as usual; 1 is the articular surface is rough, cracked, and the color is dark; 2 is the articular surface erosion, cartilage defect reaches the cartilage surface and middle layer; 3 is the articular surface ulcer formation, Cartilage defect reaches deep cartilage; 4 is divided into cartilage exfoliation and subchondral bone exposure

The rabbits' right knee articular cartilage and subchondral bone were fixed at $24 \mathrm{~h}$ with $4 \%$ polyformaldehyde solution, and paraffin slices after 10\% EDTA decalcium 15 days later, HE staining was deployed to observe the cartilage tissue structure, cell count and tidal line integrity. The scoring was based on the Mankin's method[10]. Level 0 is normal, level 1 is surface damage, level 2 is vascular palate and surface damage, level 3 is shallow fissure formation, level 4 is the fractured deep bone fissure, level 5 is a large area of deep bone cartilage deficiency, level 6 is all lost cartilage in the weight-bearing area.

\subsection{Immunohistochemistry assays}

After paraffin sections are deparaffinized and dehydrated, endogenous peroxidase was inactivated with $3 \% \mathrm{H} 2 \mathrm{O} 2$, the tissue antigen activity was repaired by trypsin digestion solution, and 1:1000 diluted mouse IL-1 $\beta$ monoclonal antibody was added and incubated at $4^{\circ} \mathrm{C}$ overnight. Rinse with PBS 3 times, $5 \mathrm{~min} /$ time. Add biotin-labeled secondary antibody dropwise, and incubate at $37^{\circ} \mathrm{C}$ for 30 minutes; rinse with PBS 3 times, $5 \mathrm{~min} /$ time. Add SABC-HRP and incubate at $37^{\circ} \mathrm{C}$ for 30 minutes. Rinse with PBS 3 times, $5 \mathrm{~min} /$ time. $\mathrm{DAB}$ develops color at room temperature, and the reaction is terminated by washing with tap water. Hematoxylin was counter-stained for 3 minutes, hydrochloric acid and alcohol were differentiated for 2 seconds, and lithium carbonate was counter-stained for 1 minute. After alcohol dehydration, the slides were mounted with gum and observed under an optical microscope. Take 5 slices in each group, use an optical microscope to magnify 200 times, randomly select 5 fields, and determine the IOD by Image-Pro Plus image analysis software.

\subsection{Fluorescence quantitative Q-PCR detection}

Take $30 \mathrm{mg}$ of cartilage and grind it into powder under liquid nitrogen. Use TRizon to extract total RNA from the tissue, and measure the absorbance (A) of the sample with an ultraviolet spectrophotometer. Reverse transcription into CDNA and PCR amplification. After amplification, the BRO-RAD gel image analysis software was used to determine the amplification curves of each group of target genes and internal controls, and calculate the difference in CT values. The primer sequences are shown in Table 1.

\subsection{Western blot analysis}


Take out cartilage tissue at $-80^{\circ} \mathrm{C}$, extract protein with RIPA kit (Biyuntian company), measure protein concentration by BCA method, Load the sample in 50ug wells. After electrophoresis on a 10\% SDS-PAGE gel, the protein was transferred to a 0.45 um pore PVDF membrane by wet transfer method, and then blocked with $5 \% \mathrm{BSA}$ at $37^{\circ} \mathrm{C}$ for 2 hours. These antibodies included goat anti-OPG (R\&D Systems, Abingdon, UK) at 1/500 dilution, rabbit anti-SOPGL (Prepotech, Neuilly-Sur-Seine, France) at 1/500, anti-IL-

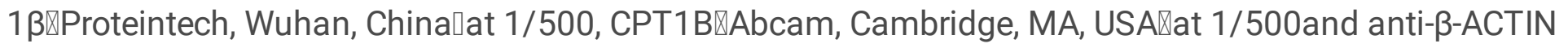
$\llbracket$ Abcam, Cambridge, MA, USA囚polyclonal antibodies (diluted 1:200), overnight at $4^{\circ} \mathrm{C}$, wash with TBST 3 times, 15 minutes each. Then apply the secondary antibody and place it at $37^{\circ} \mathrm{C}$ for 1 hour, then rinse with TBST 3 times for 15 minutes each time. Apply BeyoECL luminescent liquid in a dark room, use ChemiDoc XRS gel/luminescence image analysis system to develop protein bands, and use Quantity One software to calculate the gray value of the bands.

\subsection{Statistical analysis}

SPSS 13.0 statistical software was used for analysis. The data were expressed as mean \pm standard error of mean (SEM) unless otherwise stated. For more than a 2-group comparison, one-way ANOVA was conducted followed by a post-hoc test using Fisher's protected least significant difference. For a 2-group comparison, Student's $t$ test was conducted. The count data was used by $\chi 2$ test. Differences were considered significant when $\mathrm{P}<0.05$.

\section{Results}

\subsection{General situation of rats in each group}

No animals died during the process of membrane formation, no postoperative infections, and good health. $\mathrm{OP}+\mathrm{OA}$ and $\mathrm{OA}$ groups had painful response to local stimulation, contraction and spasm of affected limbs, obvious hindlimb claudication, weakened pedaling force, and joint instability. Abnormal mobility, disappeared after $1 w$, joint deformity and swelling after $4 w$; the external structure of the joint in the normal group was normal, no swelling, and free movement.

\subsection{Measurements of bone mineral density and bone mineral content}

We next examined any changes in BMD and BMC. Compared with the sham control, the OA and OPOA groups presented a significant reduction in $B M D$ and $B M C$ in the knee (all $P<0.05$ Figure $1 F, G$ ). Furthermore, compared with the OA group, the OPOA group showed a marked decrease in BMD and BMC.

\subsection{General observation}

The articular surface of the normal group was smooth, light blue, bright in color, no cracks or softening, no synovial hyperplasia, cool and transparent joint fluid, obvious pathological changes in the $\mathrm{OP}+\mathrm{OA}$ group, cartilage surface erosion, dark color, loss of luster, cartilage edges The hyperplasia is obvious, most of the specimens can see ulcers of varying sizes, and deep into the bone, the subchondral bone is exposed, the synovial membrane is hyperplasia and adhesion in different degrees, and the joint fluid is 
increased and cloudy. In the OA group, the cartilage surface was slightly rough, the color was light yellow, the cartilage edge had hyperplasia, some cracks and small ulcers appeared, a little hyperplasia was seen in the synovium, and the joint fluid was lightly increased.( Figure $1 \mathrm{~A}$ ), The knee cartilage degradation scores of each group are shown in Table 2.

\section{4 optical microscope observation}

The four-layer structure of articular cartilage is clear, the cartilage surface is smooth and flat, the cells in each layer are arranged neatly, and the tide line is clear; the cartilage layer of the OP+OA group is thinner, the surface is cracked, the number of cells is reduced and disordered, and the tide line is severely broken and blurred; the cartilage of the OA group. The surface is not flat, the number of cell layers increases, the arrangement is slightly disordered, and the tide line breaks into a double layer.( Figure 1B,D)

\subsection{Expression of IL-1 $\beta$ in cartilage tissue by Immunohistochemical}

The cartilage of the normal group showed a few positive cells expressing IL-1 $\beta$ on the surface; the cartilage tissue of the OP+OA group and the OA group was stained to different degrees, and the cytoplasm and nucleus of the chondrocytes were stained with yellow particles; the OP+OA group and OA group Compared with the normal group, the expression of IL-1 $\beta$ increased, and the difference was statistically significant $(P<0.05)$; compared with the OA group, the expression of IL-1 $\beta$ increased significantly in the $O P+O A$ group, and the difference was statistically significant $(P<0.05)$. (Figure $1 C, E)$

\subsection{Expression of IL-1 $1 \beta, O P G, O P G L$ and CPT1B mRNA in articular cartilage}

Compared with the normal group, the expression levels of IL-1 $\beta$, OPGL and CPT1BmRNA in the chondrocytes of the OP+OA group and the OA group were significantly increased $(p<0.05)$, (Figure $2 A, C, D$ ), while the expression content of OPGmRNA was significantly decreased $(p<0.05)$ (Figure 2 B) ;compared with OP+OA group, OPGmRNA expression level was higher in OA group $(p<0.05)$ (Figure $2 \mathrm{~B})$, IL-1 $\beta$, OPGL and CPT1BmRNA expression levels were lower $(p<0.05)$ (Figure 2A,C,D), OPG/OPGL ratio was higher in $\mathrm{OA}$ group than $\mathrm{OP}+\mathrm{OA}$ group increased, and the difference was statistically significant ( $p$ $<0.05)$. (Figure 2E)

\subsection{Expression of IL-1 $\beta$, OPG, OPGL and CPT1B protein in articular cartilage}

Compared with the normal group, the expression levels of IL-1 $\beta$, OPGL and CPT1B protein in cartilage in the $O P+O A$ group and $O A$ group were significantly increased $(p<0.05)$ (Figure $3 A, D, C)$, while the expression content of OPGmRNA was significantly decreased $(p<0.05)$ (Figure 3B),Compared with the OP+OA group, the OA group has higher OPG protein expression $(p<0.05), I L-1 \beta, O P G L, C P T 1 B$ protein expression is lower $(p<0.05)$ (Figure $3 A, B, C, D)$, and the OPG/OPGL ratio is higher in OA(Figure 3E). The difference is statistically significant $(p<0.05)$ (Figure 3E).

\section{Discussion}


The current research shows that in the OPOA model, fatty acid oxidation may be involved in the bone remodeling of subchondral bone by regulating the expression of CPT1B, thereby aggravating articular cartilage degeneration.

The result shows that OP increases the severity of cartilage damage in the knee of OA rabbit, and the pathological damage in the OPOA group was severer than that of the OA group. The major differences included changes in subchondral bone mass and pathological changes in articular cartilage. After BMD and $\mathrm{BMC}$ of the rabbit femoral head were determined, it was found that the bone mass in the OP+OA group was significantly reduced, and the cartilage destruction was severe, In this experiment, an experimental animal model was established by castration method combined with Hulth'S method. Rabbits were driven to walk for 30 minutes one week after modeling, which was equivalent to the middle and late stages of OA at 4 weeks[11]. Compared with the normal group, the Pelletier-JP and Minkin's scores of the joints in the OP+OA group and the OA group were significantly higher, indicating that the severity of the lesion is proportional to the score, and also indicating the success of this experiment. Compared with the OP+OA group, the Pelletier-JP and Minkin's scores of the OA group were reduced, indicating that the degree of joint degeneration in the OP+OA group was more severe. Compared with the OA group, the expression levels of IL-1 $\beta$ and OPGL in the OP+OA group were significantly increased, the expression of OPG was significantly decreased, and the ratio of OPG/OPGL decreased, indicating that the subchondral bone microstructure damage in osteoporotic $O A$ rabbits With remodeling intensified, it can lead to increased cartilage damage.[12] The expression of CPT1B in the OP+OA group was significantly increased, suggesting that fatty acid oxidation may be involved in the disease progression of OPOA by regulating $\mathrm{CPT} 1$.

Osteoarthritis is a disease with a high disability rate. It has become the number one killer of lost labor among the middle-aged and elderly people in my country. Its pathogenesis is closely related to cartilage cell degeneration and subchondral bone remodeling. The relationship between OP and OA is very complicated. Some studies have reported that the bone mineral density of OP and OA patients is negatively correlated with bone turnover rate [2], and there are also reports that they may have the same pathogenic mechanism. In the early stages of the disease, both OP and OA will have subchondral bone fractures or microstructure destruction[13]. The subchondral bone remodeling will accelerate the degeneration of articular cartilage. It is possible that the abnormal reconstruction of subchondral bone in the early OPOA is chiefly manifested in a change in trabecular bone[14].

The OPG/OPGL/RANK system is a key path connecting cartilage and subchondral bone. The balance of OPG and OPGL plays a decisive role in the remodeling of subchondral bone[15]. Both OPG and OPGL can be secreted by chondrocytes. When the membrane secretes protein type After OPGL binds to RANK on the surface of osteoclast precursors, it mediates the conversion of osteoclast precursors into mature osteoclasts[16]. The activation of osteoclasts promotes bone resorption, while OPG can competitively bind OPGL to inhibit bone resorption. It has been proved that a variety of cytokines that act on osteoclasts in the body are achieved by changing the ratio of OPG/OPGL[17]. In osteoblasts, OPG expression is regulated by the Wnt/ $\beta$-catenin regulatory system [6]. OPG, RANKL and RANK are also 
expressed in cartilage, but their role in chondrocyte functions is poorly understood.IL-1 $\beta$ is a key inflammatory factor in osteoarthritis, which can induce premature senescence of articular chondrocytes through multiple pathways[18]. It is suggested that the increase of IL-1 $\beta$ is positively correlated with the degree of cartilage damage[19]. Yusuke Watanabe et al. have shown that IL-1 $\beta$ can inhibit the production of osteoclasts by increasing the expression of OPG in cartilage [20].

Energy homeostasis during fasting or prolonged exercise depends on mitochondrial fatty acid oxidation (FAO).As the key rate-limiting enzyme of FAO \&carnitine palmitoyl transferase I (CPT1) regulates FAO and facilitates adaptation to the environment, both in health and disease, The CPT1 family of proteins contains three isoforms: CPT1A, CPT1B and CPT1C[21]. CPT1A and CPT1B are more similar in function although their different tissue locations provide different contexts for their physiological function. For instance, CPT1B, the muscle isoform, increases in activity and expression with exercise training . Studies have found that overexpression of CPT1 in skeletal muscle produces an increase in FAO[22]. In the skeletal muscles of thin people, the expression of CTP1B and peroxide proliferatives (PPARs) is involved in fat metabolism[23]. Although CPT1B plays an important role in human bones, and is involved in mitochondrial fatty acid oxidation, and has been shown to be differentially regulated by obesity[24]. There are clinical findings that overexpression of CPT1b enhanced fatty acid oxidation[25], and CPT1b expression increases with age to limit both intramyocellular lipid deposition and the development of insulin resistance[26]. But there has been no report that CPT1B is involved in the transcriptional regulation of bone and joints. In the report of Mo, X. B [27], CPT1 gene is related to both OA and OP, But what kind of association is not clearly. Therefore, in this experiment, by detecting the expression of CPT1B gene and protein in articular cartilage, it was found that CPT1b was differentially expressed in the control group and the experimental group, and there was a significant increase in the OPOA group. Therefore, it is necessary to consider that Mitochondrial fatty acid oxidation mediates the pathogenesis of OPOA by regulating the expression of CPT1B.

\section{Conclusion}

In summary, this study demonstrates that Mitochondrial fatty acid oxidation metabolism can be involved in the effect of osteoporosis on osteoarthritis by up-regulating the expression of CPT1b, which can mediate changes in the OPG/OPGL ratio, increase the expression of IL-1 $\beta$, and aggravate the Degeneration of OA cartilage and subchondral bone. Therefore, mitochondrial fatty acid oxidation is involved in OPOA degeneration, and further research should be done to clarify how mitochondrial fatty acid oxidation is involved in OPOA degeneration and how to treat OPOA.

\section{Abbreviations}

OA: osteoarthritis; OPG: osteoprotegerin; OPGL: osteoprotegerin ligan; CPT1B : carnitine palmitoyl transferase I B; BMD: bone mineral density; BMC: bone mineral content

\section{Declarations}


Acknowledgments

We thank the voluntary participation of each patient of this study

\section{Authors' contributions}

All authors contributed to the conception, interpretation of data, drafting/ revisions of article, provided final approval of the version to be published, and agreed to be accountable for all aspects of the work in ensuring that questions related to the accuracy or integrity of any part of the work are appropriately investigated and resolved.

\section{Funding}

This research received support from Science and Technology Commission Project of Yuzhong District, Chongqing City (No. 20170111)

\section{Availability of data and materials}

Data and material related to this study is available from the corresponding author on request.

\section{Ethics approval and consent to participate}

Ethical approval was obtained from the local university Human Research Ethics Committee『Chongqing Medical University.Volunteers meeting eligibility criteria gave informed written consent prior to enrolment by the trial administrator.

\section{Consent for publication}

Not applicable.

\section{Competing interests}

The authors certify that they have no affiliations with or financial involvement in any organization or entity with a direct financial interest in the subject matter or materials discussed in the article.

\section{References}

1. Hugle, T. and J. Geurts, What drives osteoarthritis?-synovial versus subchondral bone pathology. Rheumatology (Oxford), 2017. 56(9): p. 1461-1471.

2. Roman-Blas, J.A. and G. Herrero-Beaumont, Targeting subchondral bone in osteoporotic osteoarthritis. Arthritis Res Ther, 2014. 16(6): p. 494.

3. Li, Y.S., F.J. Zhang, C. Zeng, W. Luo, W.F. Xiao, S.G. Gao, and G.H. Lei, Autophagy in osteoarthritis. Joint Bone Spine, 2016. 83(2): p. 143-8. 
4. Zhang, J., S. Chen, W. Chen, Y. Huang, R. Lin, M. Huang, Y. Wu, L. Zheng, Z. Li, N. Liao, J. Ye, and X. Liu, Ultrastructural change of the subchondral bone increases the severity of cartilage damage in osteoporotic osteoarthritis of the knee in rabbits. Pathol Res Pract, 2018. 214(1): p. 38-43.

5. Nagy, E.E., T. Varga-Fekete, A. Puskas, P. Kelemen, Z. Brassai, K. Szekeres-Csiki, T. Gombos, M.C. Csanyi, and J. Harsfalvi, High circulating osteoprotegerin levels are associated with non-zero blood groups. BMC Cardiovasc Disord, 2016. 16(1): p. 106.

6. Kovacs, B., E. Vajda, and E.E. Nagy, Regulatory Effects and Interactions of the Wnt and OPG-RANKLRANK Signaling at the Bone-Cartilage Interface in Osteoarthritis. Int J Mol Sci, 2019. 20(18).

7. Liu, K., L.J. Tan, P. Wang, X.D. Chen, L.H. Zhu, Q. Zeng, Y. Hu, and H.W. Deng, Functional relevance for associations between osteoporosis and genetic variants. PLoS One, 2017. 12(4): p. e0174808.

8. Castaneda, S., E. Calvo, R. Largo, R. Gonzalez-Gonzalez, C. de la Piedra, M. Diaz-Curiel, and G. Herrero-Beaumont, Characterization of a new experimental model of osteoporosis in rabbits. J Bone Miner Metab, 2008. 26(1): p. 53-9.

9. Pelletier, J.P., D. Jovanovic, J.C. Fernandes, P. Manning, J.R. Connor, M.G. Currie, J.A. Di Battista, and J. Martel-Pelletier, Reduced progression of experimental osteoarthritis in vivo by selective inhibition of inducible nitric oxide synthase. Arthritis Rheum, 1998. 41(7): p. 1275-86.

10. Ryu J, T.B., Mankin HJ., Ryu J, Treadwell BV, Mankin HJ. Biochemical and metabolic abnormalities in normal and osteoarthritic human articular cartilage. Arthritis Rheum. 1984,27(1):49-57. doi:10.1002/art.1780270109. Arthritis Rheum. .

11. Chen, S., Y. Huang, W. Chen, G. Wu, N. Liao, X. Li, M. Huang, R. Lin, C. Yu, X. Li, and X. Liu, Protective effects of the Tougu Xiaotong capsule on morphology and osteoprotegerin/nuclear factor-kappaB ligand expression in rabbits with knee osteoarthritis. Mol Med Rep, 2016. 13(1): p. 419-25.

12. Bellido, M., L. Lugo, J.A. Roman-Blas, S. Castaneda, J.R. Caeiro, S. Dapia, E. Calvo, R. Largo, and G. Herrero-Beaumont, Subchondral bone microstructural damage by increased remodelling aggravates experimental osteoarthritis preceded by osteoporosis. Arthritis Res Ther, 2010. 12(4): p. R152.

13. lijima, H., T. Aoyama, J. Tajino, A. Ito, M. Nagai, S. Yamaguchi, X. Zhang, W. Kiyan, and H. Kuroki, Subchondral plate porosity colocalizes with the point of mechanical load during ambulation in a rat knee model of post-traumatic osteoarthritis. Osteoarthritis Cartilage, 2016. 24(2): p. 354-63.

14. Zheng, W., B. Ding, X. Li, D. Liu, H. Yokota, and P. Zhang, Knee loading repairs osteoporotic osteoarthritis by relieving abnormal remodeling of subchondral bone via Wnt/beta-catenin signaling. FASEB J, 2020. 34(2): p. 3399-3412.

15. Chen, D., Y. Liu, Z. Liu, and P. Wang, OPG is Required for the Postnatal Maintenance of Condylar Cartilage. Calcif Tissue Int, 2019. 104(4): p. 461-474.

16. Corrado, A., A. Neve, A. Macchiarola, A. Gaudio, A. Marucci, and F.P. Cantatore, RANKL/OPG ratio and DKK-1 expression in primary osteoblastic cultures from osteoarthritic and osteoporotic subjects. J Rheumatol, 2013. 40(5): p. 684-94.

17. Cafferata, E.A., G. Monasterio, F. Castillo, P. Carvajal, G. Flores, W. Díaz, A.D. Fuentes, and R. Vernal, Overexpression of MMPs, cytokines, and RANKL/OPG in temporomandibular joint osteoarthritis and 
their association with joint pain, mouth opening, and bone degeneration. A preliminary report. Oral Dis, 2020.

18. Speichert, S., N. Molotkov, K. El Bagdadi, A. Meurer, F. Zaucke, and Z. Jenei-Lanzl, Role of Norepinephrine in IL-1 $\beta$-Induced Chondrocyte Dedifferentiation under Physioxia. International Journal of Molecular Sciences, 2019. 20(5).

19. Fei, J., B. Liang, C. Jiang, H. Ni, and L. Wang, Luteolin inhibits IL-1beta-induced in fl ammation in rat chondrocytes and attenuates osteoarthritis progression in a rat model. Biomed Pharmacother, 2019. 109: p. 1586-1592.

20. Watanabe, Y., A. Namba, Y. Aida, K. Honda, H. Tanaka, N. Suzuki, H. Matsumura, and M. Maeno, IL1 beta suppresses the formation of osteoclasts by increasing OPG production via an autocrine mechanism involving celecoxib-related prostaglandins in chondrocytes. Mediators Inflamm, 2009. 2009: p. 308596.

21. Schlaepfer, I.R. and M. Joshi, CPT1A-mediated Fat Oxidation, Mechanisms, and Therapeutic Potential. Endocrinology, 2020. 161(2).

22. Alsanea, S., M. Gao, and D. Liu, Phloretin Prevents High-Fat Diet-Induced Obesity and Improves Metabolic Homeostasis. Aaps j, 2017. 19(3): p. 797-805.

23. Araki, M., Y. Nakagawa, A. Oishi, S.I. Han, Y. Wang, K. Kumagai, H. Ohno, Y. Mizunoe, H. Iwasaki, M. Sekiya, T. Matsuzaka, and H. Shimano, The Peroxisome Proliferator-Activated Receptor a (PPARa) Agonist Pemafibrate Protects against Diet-Induced Obesity in Mice. Int J Mol Sci, 2018. 19(7).

24. Bétry, C., E. Meugnier, M. Pflieger, G. Grenet, S. Hercberg, P. Galan, E. Kesse-Guyot, H. Vidal, and M. Laville, High expression of CPT1b in skeletal muscle in metabolically healthy older subjects. Diabetes Metab, 2019. 45(2): p. 152-159.

25. Bruce, C.R., A.J. Hoy, N. Turner, M.J. Watt, T.L. Allen, K. Carpenter, G.J. Cooney, M.A. Febbraio, and E.W. Kraegen, Overexpression of carnitine palmitoyltransferase-1 in skeletal muscle is sufficient to enhance fatty acid oxidation and improve high-fat diet-induced insulin resistance. Diabetes, 2009. 58(3): p. 550-8.

26. Chee, C., C.E. Shannon, A. Burns, A.L. Selby, D. Wilkinson, K. Smith, P.L. Greenhaff, and F.B. Stephens, Relative Contribution of Intramyocellular Lipid to Whole-Body Fat Oxidation Is Reduced With Age but Subsarcolemmal Lipid Accumulation and Insulin Resistance Are Only Associated With Overweight Individuals. Diabetes, 2016. 65(4): p. 840-50.

27. Mo, X.B., X. Lu, Y.H. Zhang, Z.L. Zhang, F.Y. Deng, and S.F. Lei, Gene-based association analysis identified novel genes associated with bone mineral density. PLoS One, 2015. 10(3): p. e0121811.

\section{Tables}


Table 1

Primer Sequence

\begin{tabular}{|c|c|c|c|}
\hline GENE & primer & Sequence $\left(5^{\prime}->3^{\prime}\right)$ & Product length \\
\hline \multirow[t]{2}{*}{ OPG } & Forward & CACGTCAAGCAGGAGTGCAA & 123 \\
\hline & Reverse & TTGTGCCACTCCAAACCCAG & 123 \\
\hline \multirow[t]{2}{*}{ IL-1 $\beta$} & Forward & TCTGTGACTCGTGGGATGAT & 69 \\
\hline & Reverse & CTTCTTTGGGTATTGTTTGG & 69 \\
\hline \multirow[t]{2}{*}{ ACTIN } & Forward & CTAACGGCGCAGAAACGAGA & 186 \\
\hline & Reverse & TCGGCCACATTGCAGAACTT & 186 \\
\hline \multirow[t]{2}{*}{ CPT1b } & Forward & AAGCGACCTCCAGGATCTCTTC & 159 \\
\hline & Reverse & AGAGCACCTCAGCCAGGAACG & 159 \\
\hline \multirow[t]{2}{*}{ OPGI } & Forward & ACGCCAACATTTGCTTTCGC & 116 \\
\hline & Reverse & GAGGGTGTGAGAGCTTGGGA & 116 \\
\hline
\end{tabular}

Table 2

Grading of knee cartilage degeneration in each group (n)

\begin{tabular}{|lllllllll|}
\hline \multirow{2}{*}{ Group } & Specimen & \multicolumn{3}{l}{ Rating } & \multicolumn{4}{l}{ AverageRidit value } \\
\cline { 3 - 6 } & & L0 & L1 & L2 & L3 & L4 & \\
\hline OA & 10 & 0 & 4 & 4 & 2 & 0 & $0.685^{\star \#}$ \\
\hline OP+OA & 10 & 0 & 0 & 1 & 3 & 6 & $0.447^{*}$ \\
Normal control & 10 & 10 & 0 & 0 & 0 & 0 & 0.833 \\
Totol & 30 & 10 & 4 & 5 & 5 & 6 & 0.5 \\
\hline
\end{tabular}

${ }^{\star} \mathrm{P}<0.05,{ }^{\star *} \mathrm{P}<0.01$ and ${ }^{* \star *} \mathrm{P}<0.001$ vs sham group; $\# \mathrm{P}<0.05$, \#\#P $<0.01$ and \#\#\#P $<0.001$ vs $\mathrm{OP}+\mathrm{OA}$ groups;

Figures 


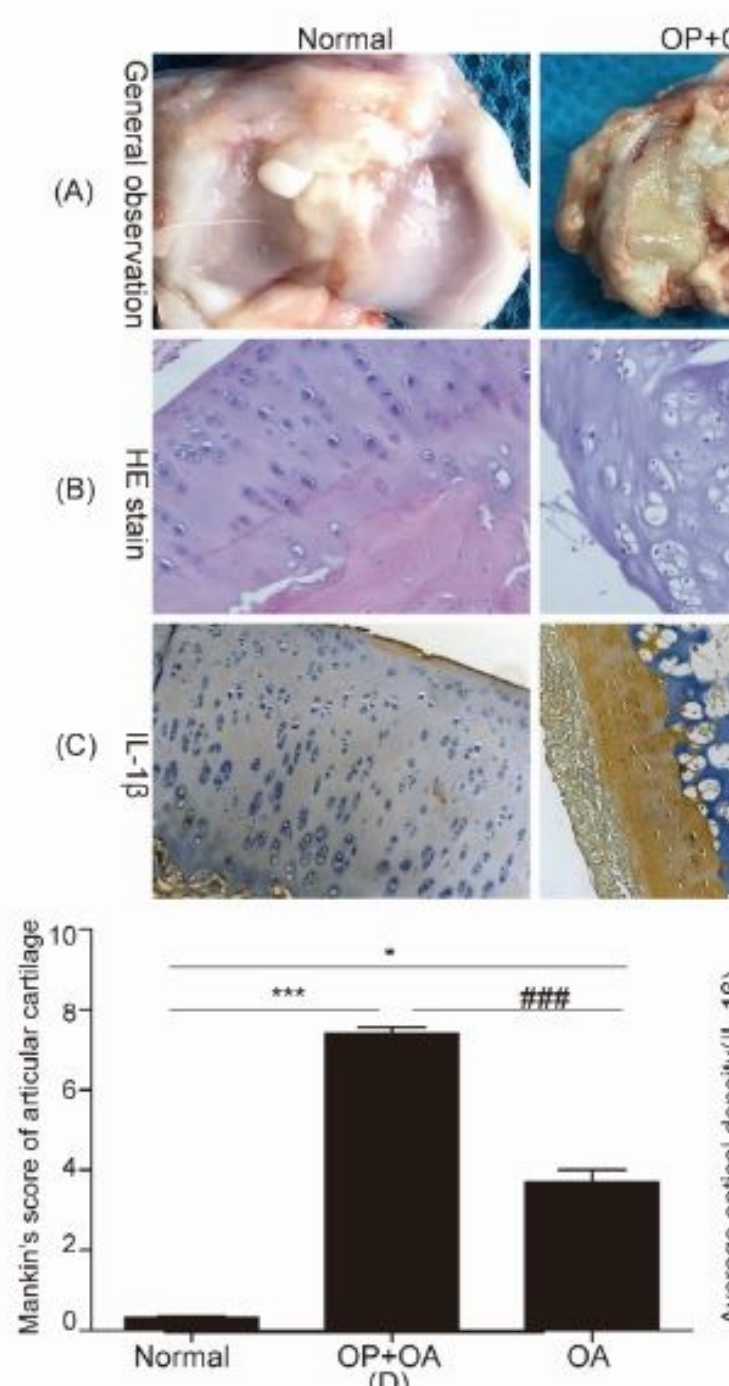

(D)



(F)

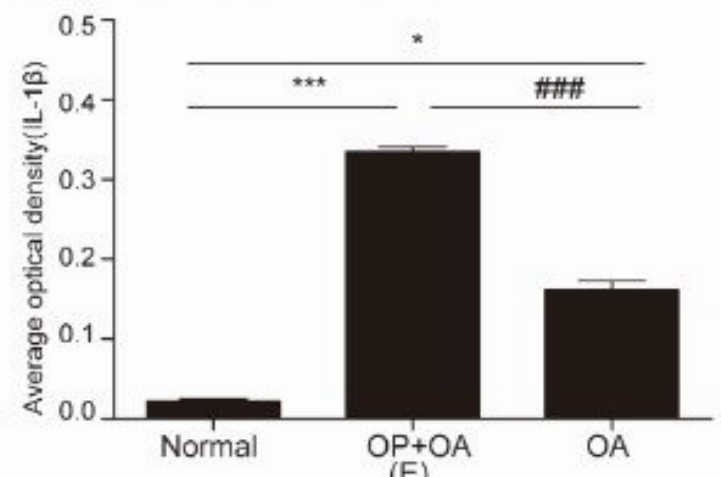

(E)

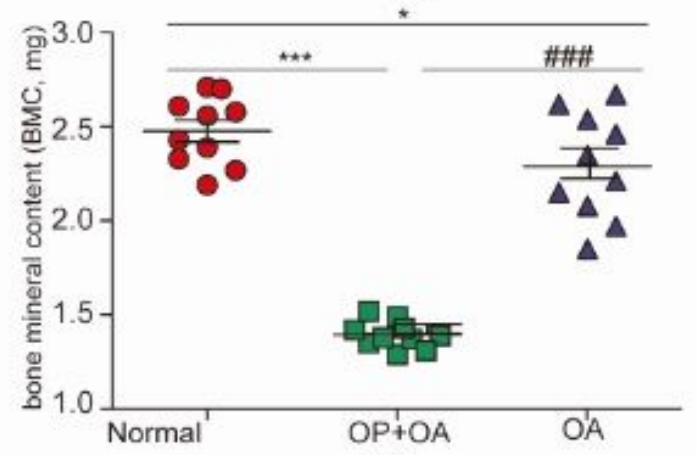

(G)

\section{Figure 1}

(A) (B) General observation and Hematoxylin-eosin staining of knee joint sections in the control group showed a smooth surface and a clear laminar structure in the articular cartilage. By contrast, the cartilage surface in the OP+OA group was rough with some superficial leakage and ulcers. Furthermore, OA group cartilage showed fewer lesions compared with the OP+OA group (magnification,200x). (C) Immunohistochemical staining of IL-1 $\beta$ (magnification,400x) using image analysis of Image-ProPlus7.0. 
(D) Mankin's score of articular cartilage. Data are expressed as mean \pm SD. (E) Data are expressed as mean \pm SDF and G, BMD and BCM detection of rabbit right femur. $* p<0.05,{ }^{\star \star} p<0.01$, ${ }^{\star \star \star} p<0.001$

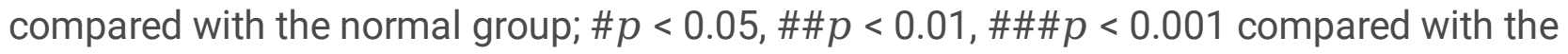
OP+OAgroups $\$

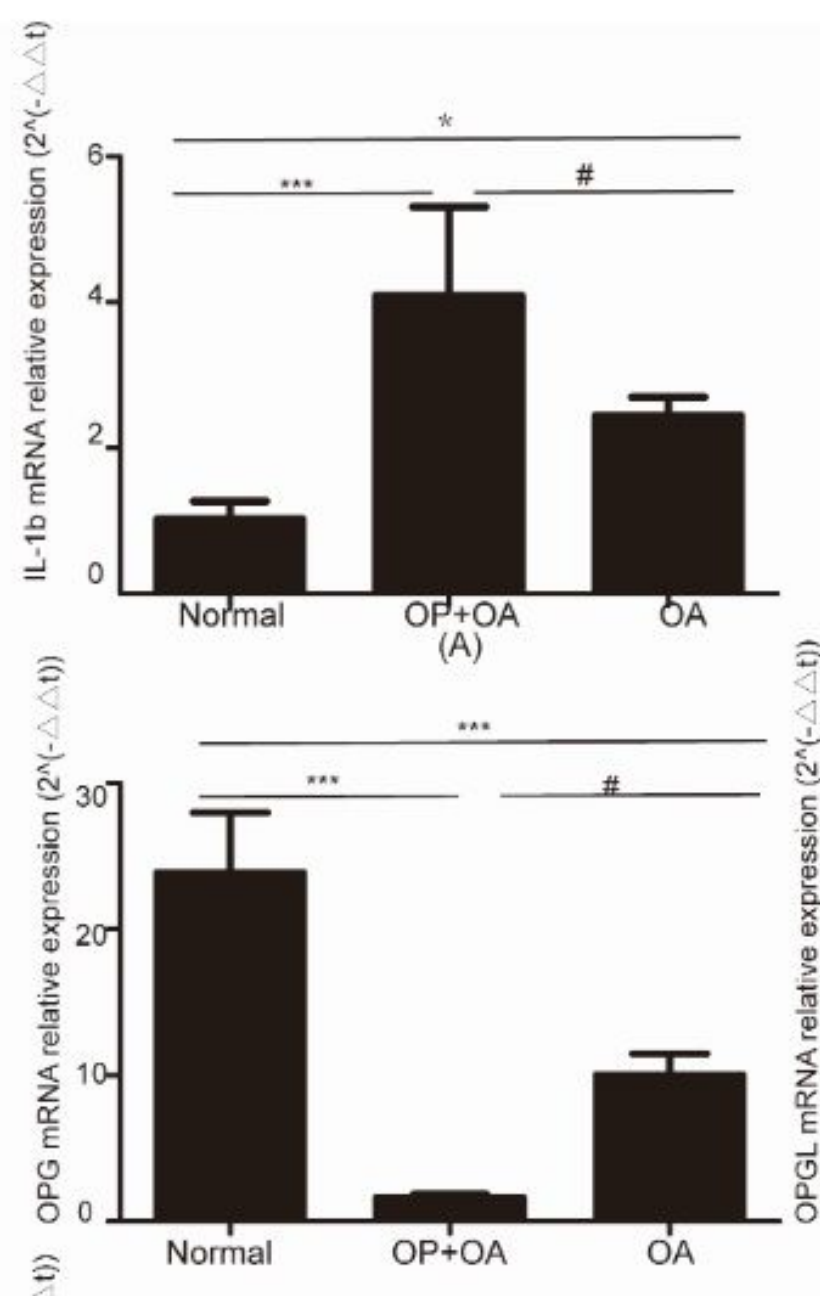

(B)

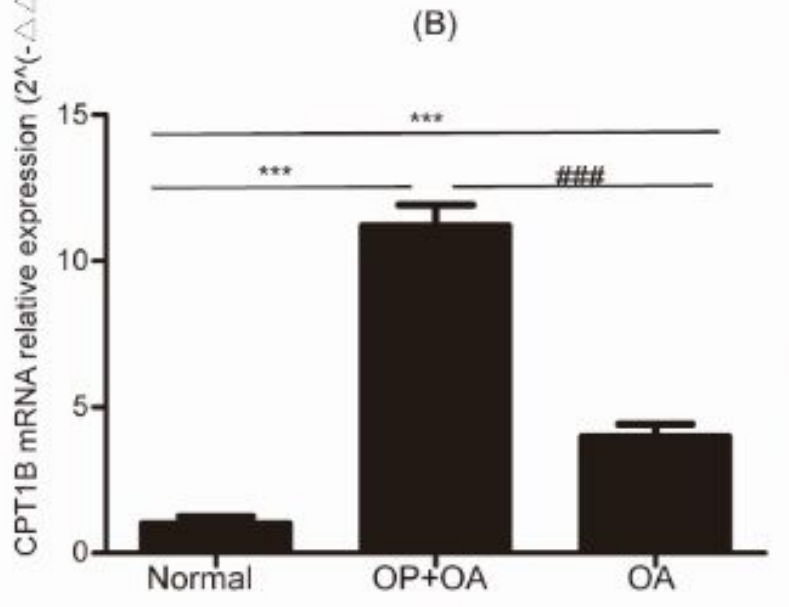

(D)

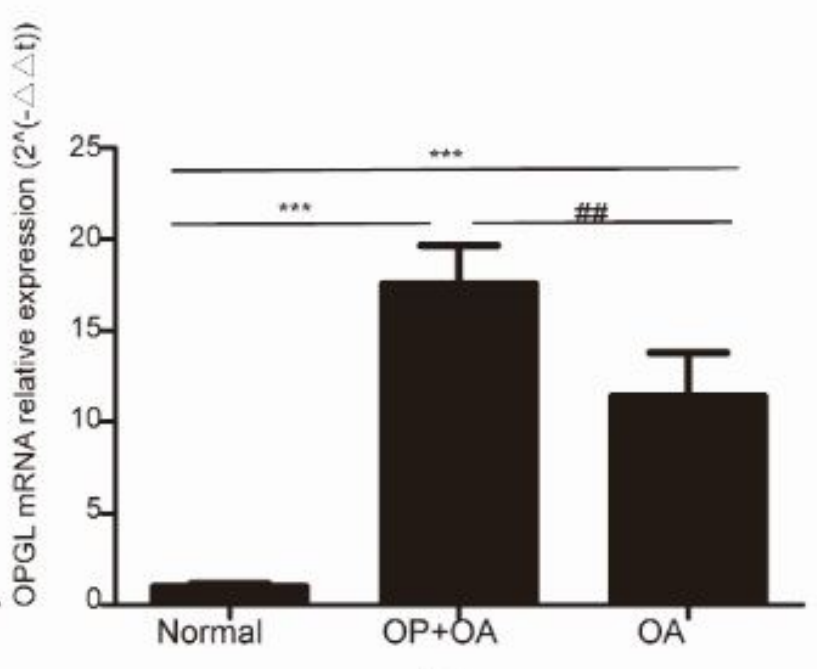

(C)

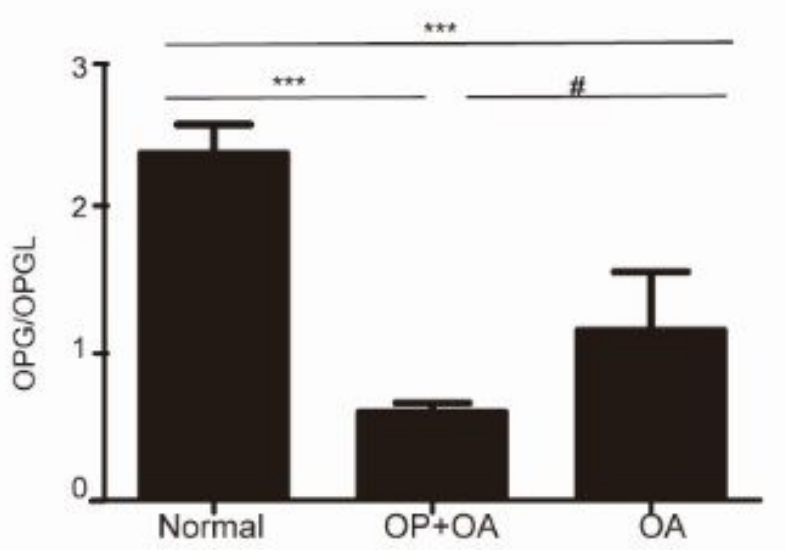

(E)

Figure 2 
Expression of IL-1 $\beta$, OPG, OPGL and CPT1b mRNA in articular cartilage. (A), (B), (C), and (D), respectively, showed that IL-1 $\beta, O P G, O P G L$ and $C P T 1 b$ mRNA were detected by quantitative real-time polymerase chain reaction. (E) Represents the ratio of OPG/OPGL. Data are expressed as mean $\pm \mathrm{SD}$ (each group,

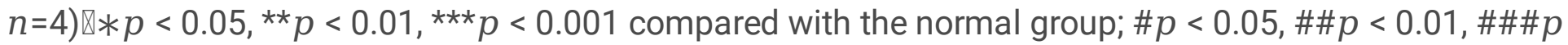
$<0.001$ compared with the OP+OAgroups $\rrbracket$


(B)

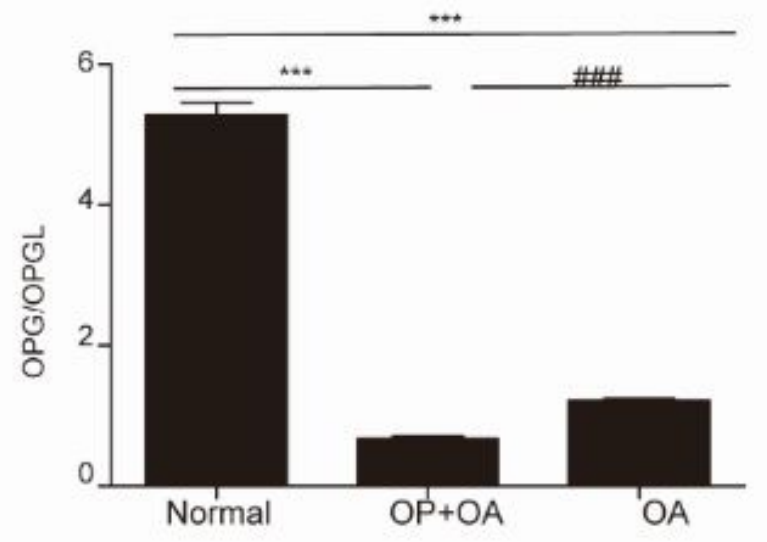

(E)

Figure 3 
Western blot showed the protein level of I IL-1 3, OPG, OPGL and CPT1B and relative quantification of protein level of IL-1 $\beta \bowtie A \rrbracket, O P G(B), O P G L(D)$ and CPT1B(C). (E) Represents the ratio of OPG/OPGL. Data are expressed as mean $\pm \mathrm{SD}$ (each group, $n=4$ ) $\varangle * p<0.05$, ${ }^{* *} p<0.01$, ${ }^{* \star *} p<0.001$ compared with the

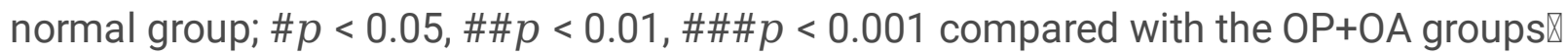

